\title{
A Qualitative Investigation of the Impact of Acne on Health-Related Quality of Life (HRQL): Development of a Conceptual Model
}

\author{
Gabriella Fabbrocini - Sara Cacciapuoti - Giuseppe Monfrecola
}

Received: November 8, 2017 / Published online: February 12, 2018

(C) The Author(s) 2018. This article is an open access publication

\begin{abstract}
Introduction: The negative impact of acne on aspects of health-related quality of life (HRQL) has been demonstrated in many quantitative studies; however, there has been relatively little qualitative research exploring the impact of acne and the use of topical treatment. The study aimed to explore the impact of moderate-severe acne on HRQL in adolescents and adults with inflammatory and non-inflammatory lesions and to develop a conceptual model to illustrate the impact. In addition, the study aimed to identify the attributes of topical acne treatments that are most important for patients.

Methods: Thirty-four adolescents and 16 adults with moderate-severe acne who were currently/ recently prescribed topical treatment were recruited in this cross-sectional qualitative study in the UK, Italy, and Germany. In-depth, semi-structured telephone interviews explored patients' experiences of acne and the impact it
\end{abstract}

Enhanced content To view enhanced content for this article go to https://doi.org/10.6084/m9.figshare. 5817453

G. Fabbrocini $(\bowtie) \cdot$ S. Cacciapuoti $\cdot$ G. Monfrecola Section of Dermatology, Department of Clinical Medicine and Surgery, University of Naples Federico II, Naples, Italy

e-mail: gafabbro@unina.it has on their HRQL, and their experience of topical treatments for acne. Data were analyzed using thematic analysis and a conceptual model was developed.

Results: The analysis identified seven main areas of HRQL that are affected by acne: emotional functioning, social functioning, relationships, leisure activities, daily activities, sleep, and school/work. Also common throughout the interviews was the perception and reaction to acne from others, which potentially had an impact on all areas of HRQL. The conceptual model illustrates the impact on HRQL and the links between HRQL domains. For both adolescents and adults, it was most important for acne treatments to be fast-acting, non-irritating, and non-bleaching.

Conclusion: The results of this qualitative study demonstrate that moderate-severe acne has an extensive impact on adolescents' and adults' HRQL. The conceptual model illustrates the many areas of HRQL that are affected and draws attention to the importance of effective treatments for acne. The study also highlights topical acne treatment attributes that are most important for patients.

Funding: Meda, a Mylan company.

Keywords: Acne vulgaris; Conceptual model; Health-related quality of life; Qualitative; Topical treatment 


\section{INTRODUCTION}

Acne is a chronic, inflammatory disease of the pilosebaceous unit estimated to affect $9.4 \%$ of the global population [1]. By the age of 21 years, $80-90 \%$ of people are estimated to have had acne [2]. The primary pathogenic factors are increased sebum production by the sebaceous gland, alterations in the keratinization process, follicular colonization by Propionibacterium acnes, and activation of innate immunity followed by increased inflammation [3]. Signs of acne include non-inflammatory lesions (open and closed comedones), inflammatory lesions (papules and pustules), and seborrhea (oily skin). Typically, acne starts in early puberty and is a chronic condition that can last for many years [4].

Although acne is not life-threatening or physically disabling, the negative impact of acne on health-related quality of life (HRQL) has been demonstrated in many studies of adults and adolescents with acne [5-7]. In one study, pretreatment acne patients reported substantially more pain/discomfort and anxiety/depression than a population sample [5]. In another study, authors claimed that the HRQL of acne patients is comparable to patients with conditions such as chronic disabling asthma, epilepsy, back pain, and arthritis [6]; this is supported by a literature review that found the HRQL impact of acne was comparable to psoriasis, a condition that causes significant disability [8]. A small study of adults with acne found clinically significant anxiety and depression in $44 \%$ and $18 \%$ of the sample, respectively [9], and a large survey of 18-year-olds found that participants with acne had significantly more depressive symptoms, lower self-attitude and self-worth, more feelings of uselessness, and lower body satisfaction than those without acne [10]. Other psychological effects of facial acne, including embarrassment, impaired self-image, low self-esteem, self-consciousness, frustration, and anger, have been identified in qualitative research [11]. In addition, dermatological-related social anxiety has been shown to be negatively associated with intention to participate in sport/exercise, self-esteem, and dermatological HRQL [12]. A review of studies examining the relationship between HRQL and acne in adolescents concluded that acne has a negative impact on HRQL and improvements in acne are associated with improvement in individuals' self-appraisals, thus highlighting the potential benefit of effective acne treatments on HRQL [13].

Many treatments for acne are currently available; guidelines recommend a combination of a topical retinoid and an antimicrobial agent for most patients with acne in order to target both inflammatory and non-inflammatory lesions [14]. Although acne typically requires prolonged treatment, poor adherence to acne therapies has been documented. One large study found poor adherence in 50\% of participants prescribed treatment for acne; adherence rates reported in Europe were lower than those reported in the Americas or Asia [15]. Dissatisfaction with treatment has been closely associated with poor adherence [16]. Understanding more about what drives treatment satisfaction and what patients prefer in terms of their acne treatment may be important. A conjoint analysis assessing patient preferences for topical therapies evaluated five treatment attributes (form, storage, product life, method of application, and regimen). After using four topical treatments for a week each, participants preferred a gel formulation, room temperature storage, application with fingers, and a oncedaily regimen [17].

Much of the previous research into the impact of acne on HRQL has used validated instruments such as the Acne Quality of Life (Acne-QOL) [18], Dermatology Quality of Life Index (DLQI) [19], or the Assessment of Quality of Life (A-QOL) [20]. Some qualitative studies have explored the psychological and social impact of acne [11, 21, 22]. Qualitative research methodologies allow an in-depth investigation into patients' experiences and allow us to identify concepts of importance to patients, in terms of HRQL impact and experience of using treatment for acne. Qualitative data can be used to develop conceptual models to illustrate the impact of a condition and hypothesize links between the concepts. A conceptual model can be used to guide the choice of what to measure 
and how to measure it, and provide a context for the interpretation of findings [23].

The aims of this qualitative study were to explore the impact of moderate-severe acne on HRQL in adolescents and adults with inflammatory and non-inflammatory lesions, and to develop a conceptual model to illustrate this impact. The study also aimed to determine the attributes of topical treatments for acne that are the most important for patients.

\section{METHODS}

\section{Study Design}

Qualitative methods were used to explore the impact of moderate-severe acne on adolescents' and adults' quality of life. Individual interviews were conducted with acne patients to inform the development of a conceptual model of patient-reported HRQL. The research was designed to comply with guidelines for conducting research with children and young people [24]. Ethical approval was provided by Salus IRB, an independent review board, and the study was conducted in accordance with the principles of the Declaration of Helsinki. All adult participants provided informed consent prior to participating in the study; parents or guardians of adolescent participants provided informed consent for their child to participate; adolescents also provided assent.

\section{Participants}

Interview participants were recruited through a specialist recruitment panel in the UK, Italy, and Germany. Participants were eligible if they had a self-reported diagnosis of acne vulgaris, were aged 12-17 years (adolescents) or 18 years or older (adults), were currently experiencing facial lesions including papules and/or pustules, and were currently or recently (in the last 6 months) prescribed a topical medication for acne. Recruitment aimed for a higher proportion of adolescents than adults in each country to reflect the higher incidence in younger people.

\section{Data Collection Procedures}

Semi-structured interview guides were developed following a literature review of HRQL in acne; separate versions were developed for adult and adolescent interviews. The interviews began with a series of sociodemographic and clinical questions followed by a semi-structured interview guide exploring patients' experience of acne. The interview guide used open-ended questions to allow participants to spontaneously describe the ways in which acne affects them, e.g., "what is the most difficult part of having acne?", followed by open-ended questions about different areas of HRQL. If not already reported, specific probe questions were also asked such as "how does acne affect your self-confidence or self-esteem?" Interviews also explored participants' experiences of using topical treatment for acne and their views on different aspects of topical therapy. The interview guides were modified during the study to incorporate questions on topics mentioned in early interviews. Interviews were conducted by telephone; evidence suggests that telephone interviews can be used productively in qualitative research, including research with children, with no significant differences in the quality of the data obtained when compared with face-toface interviews $[25,26]$. Telephone interviews are also particularly suitable for research on dermatological conditions as they allow participants to speak freely about their acne without feeling self-conscious that the interviewer can see their acne. The telephone interviews were conducted by experienced qualitative researchers following semi-structured adolescent or adult interview guides. Interviews lasted up to an hour and were audio recorded and transcribed verbatim.

\section{Analysis}

Data from the interviews were analyzed using thematic analysis, which uses inductive coding to identify themes across a dataset [27]. Transcripts were systematically coded using a qualitative software tool (MAXQDA). A coding framework was developed and discussed by the 
study team, which was then used throughout the analysis. Transcripts from adolescent and adult interviews were grouped and analyzed separately to allow for identification of concepts or themes occurring in only one group. Saturation, the point at which no new information is obtained from additional interviews [28], was assessed using saturation tables; the study continued until saturation was reached. The conceptual model was developed using the concepts and themes identified in the analysis. The model was reviewed by a dermatologist and revised until all authors were in agreement.

\section{RESULTS}

\section{Sample Demographics}

Fifty participants were recruited and interviewed; 34 adolescents and 16 adults with moderate-severe acne from the UK $(N=20$ adolescents and $N=10$ adults), Italy $(N=7$ adolescents and $N=3$ adults), and Germany ( $N=7$ adolescents and $N=3$ adults). The demographics of the sample are shown in Table 1. Over half of the adolescent sample was female and three quarters identified themselves as white, with an mean age of 15 years. The adult sample had a mean age of 28 years; however, the UK sample was older on average (mean age 32 years) than the Italian (mean age 23 years) and German (mean age 19 years) samples. The adult sample had an equal number of male and female participants. Most of the sample rated their acne as at least moderate at the time of the interview and at least severe when at its worst.

\section{Qualitative Results}

For most participants, acne had an impact on many areas of their HRQL; however, one adolescent participant reported no impact on his HRQL. The analysis identified seven main areas of HRQL that are affected by acne: emotional functioning, social functioning, relationships, leisure activities, daily activities, and impact on sleep and school/work. The perception of acne from others and their reaction to it was also a common concept. Figure 1 shows the percentage of adolescents and adults reporting an impact on each of the domains, demonstrating that for almost all domains, a higher proportion of adult participants reported an impact compared with adolescents. Each of the domains and their inter-relationships are described in the sections below, with example quotations provided in Figs. 2 and 3.

\section{Emotional Functioning}

All except one adolescent reported that acne had a detrimental impact on some aspect of their emotional well-being, particularly on their self-confidence or self-esteem. For some participants, this was due to reaction from others. Having less confidence impacted other areas of participants' lives, such as participation in school activities and socializing. Two-thirds of adolescents reported that acne made them feel down and almost half of participants felt lonely or isolated. Half of adolescents also felt frustrated by their acne because of having no control over it or treatments not working.

Adult participants reported similar emotional impacts; almost all felt that acne lowered their self-confidence or self-esteem, made them feel down or depressed, and self-conscious or embarrassed. Adults also reported feeling isolated or lonely because of their acne.

\section{Social Functioning}

Over two-thirds of adolescent and all adult participants reported that acne had an impact on their social activities. In particular, participants mentioned that interacting with strangers is difficult; they felt that people focused on their acne when speaking to them or judged them because of their acne. Participants did not want to socialize when their acne flared up and some participants did not attend activities if it meant that they would not be able to wear makeup.

Most participants reported that acne affected their use of social media. This included not posting pictures of themselves if their acne was visible, editing photos before posting them, 


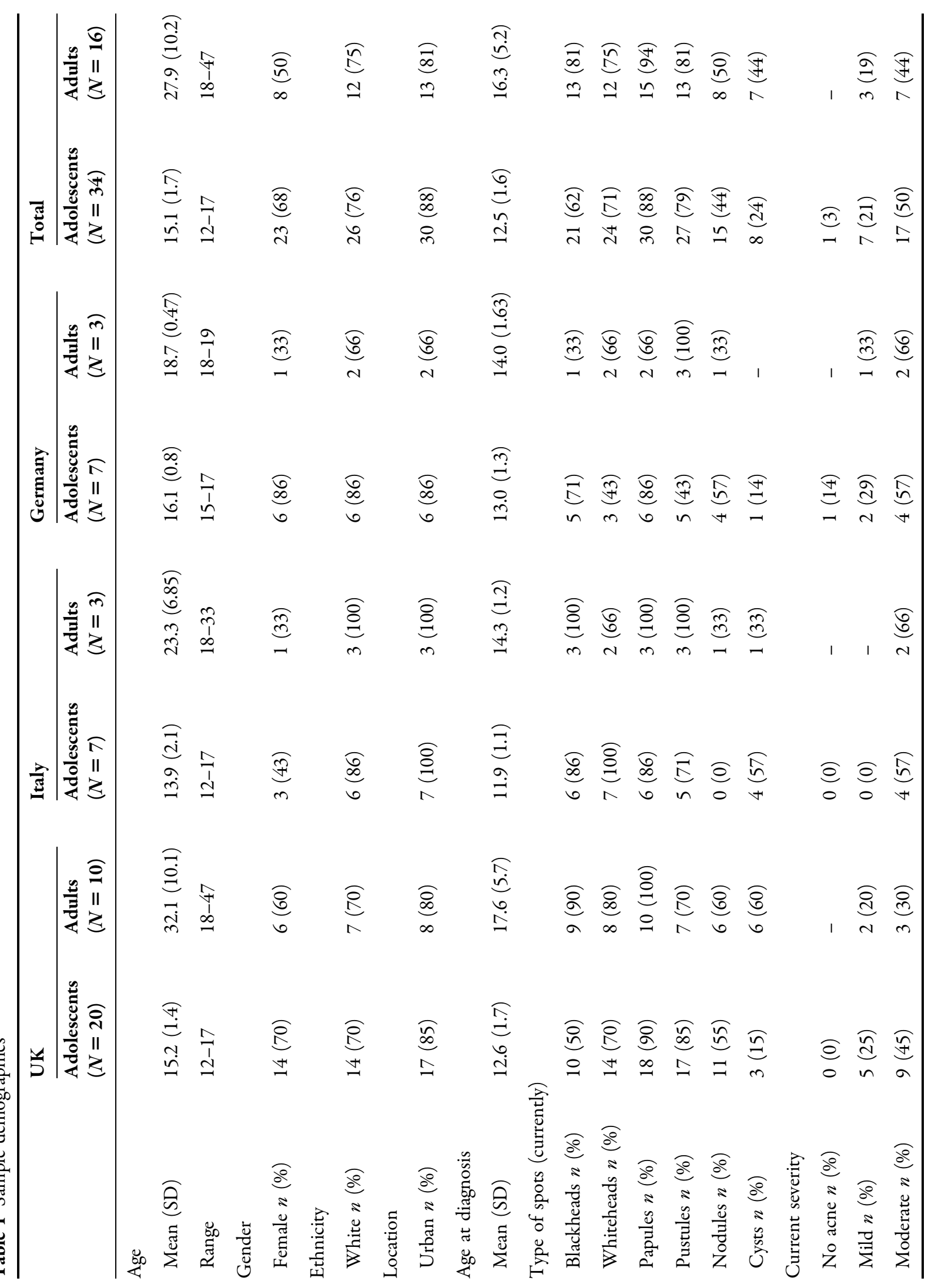




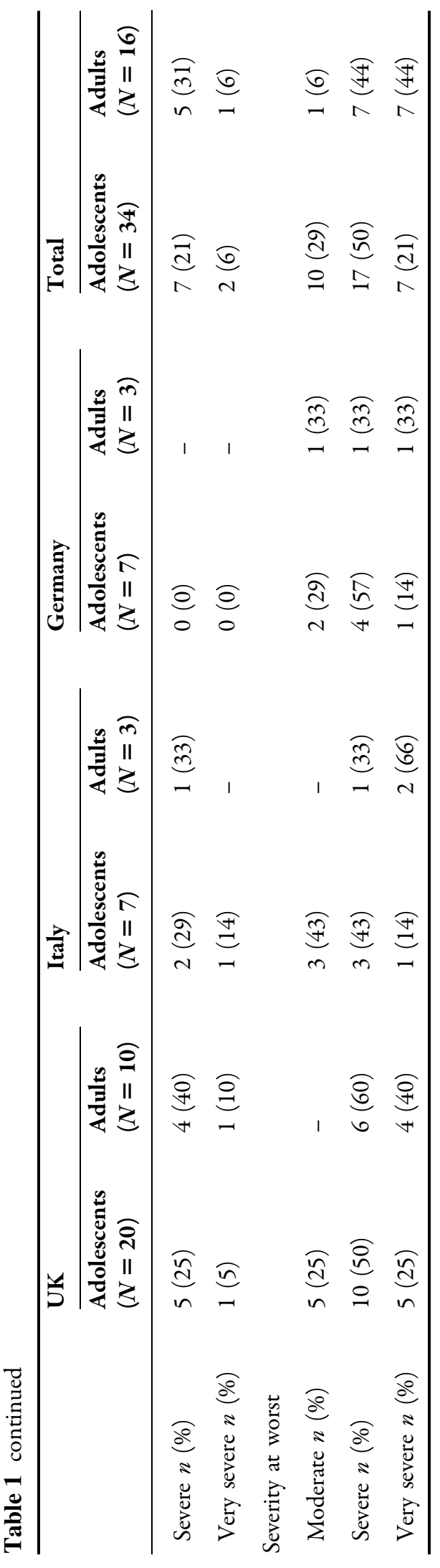

asking people to remove photos of them, avoiding being in photos, or making sure they were in the background of group pictures.

\section{Relationships}

Almost half of adolescents and several adults reported that acne has an impact on them when talking to unfamiliar people. For some this was due to a lack of confidence, while others worried how new people would perceive their acne. Participants described feeling "discomfort", "embarrassed", "anxious", or "intimidated" when talking to new people. Some adolescents reported that acne has an impact on forming relationships with girlfriends/boyfriends, for the same reasons. Two adult participants mentioned that their acne had caused relationships to end. Some adolescents also discussed problems acne caused with their friendships; this included friends making fun of their acne or friends not understanding when they complain about their acne. Almost half of the adolescent sample had friends with acne, which meant that they found it easier to cope with their own acne. In contrast, several adults did not have any friends with acne and therefore felt they did not have anyone to talk to who understood.

\section{Leisure Activities}

The leisure activities most commonly affected by acne were swimming and sports/exercise. Participants did not like to go swimming because other people would see more of their acne; some felt that chlorine would aggravate their acne, others did not want to be seen without makeup, and some adults did not want their acne scars to be visible. Taking part in sport was also affected by acne for several reasons; some participants found that their acne was made worse by sweat, while others did not want to join teams with strangers as they felt they would stare at their acne. Participants also reported feeling self-conscious about their acne when getting changed or when wearing an athletics vest. Four adolescents discussed acne having an impact on them taking part in activities such as acting or public speaking 


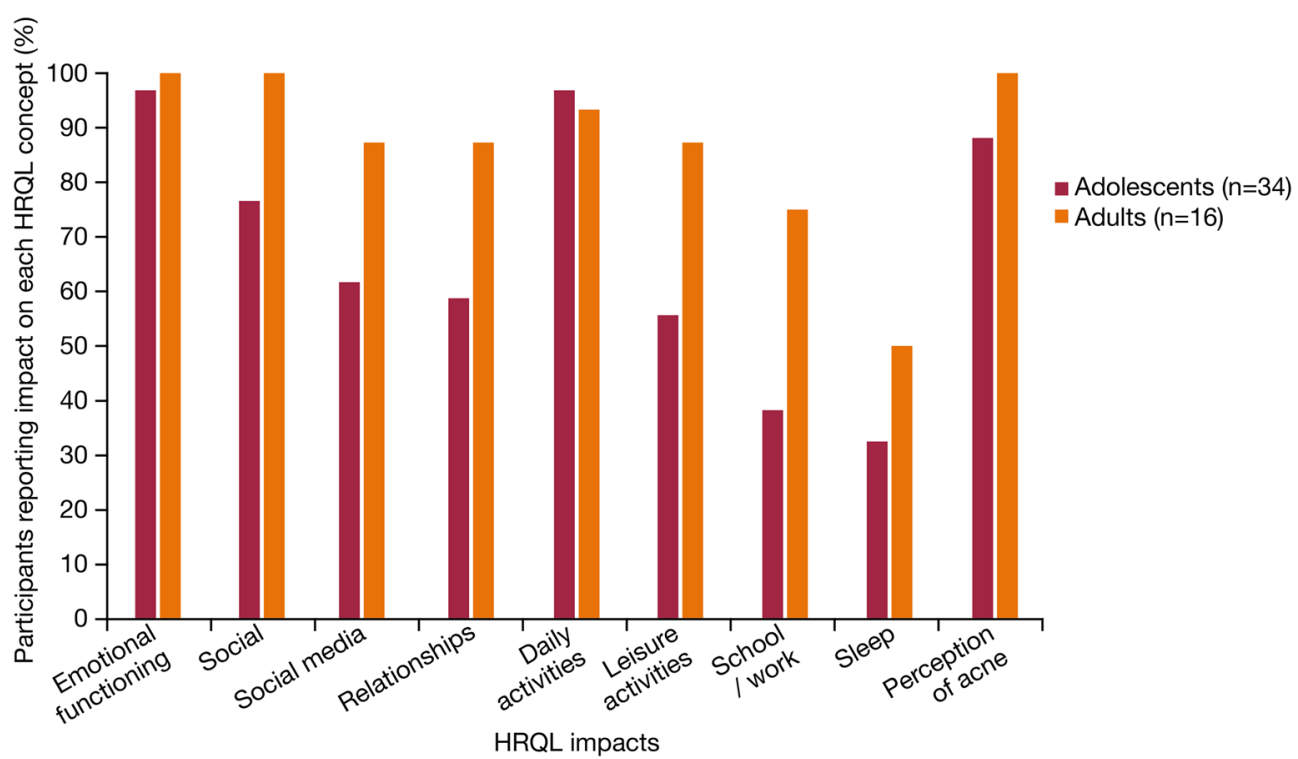

Fig. 1 Percentage of adolescent $(n=34)$ and adult $(n=16)$ participants reporting an impact of acne on each health-related quality of life (HRQL) concept. This figure was first presented at the 26th European Academy

because they did not have the confidence to stand up in front of others.

\section{Daily Activities}

Both adolescents and adults discussed the timeconsuming aspect of acne, having to allow time for their skin care and treatment routine each day. All female participants discussed makeup; a few did not wear cosmetics as they felt it would make their acne worse, others used makeup to cover up their acne and make it less noticeable. Some would not leave their house without makeup on. Some participants did not wear certain clothes because of their acne, as they did not want their shoulders or chest to be visible or they did not want new clothes to be bleached by their acne medication. Participants would feel anxious or embarrassed and feel like people were staring at them; therefore, they avoided going out when their acne was at its worst.

\section{Impact on Sleep}

Acne had an impact on sleep for a third of adolescent and half of adult participants. For of Dermatology and Venereology Congress, September 13-17, 2017, Geneva, Switzerland

several this was due to pain or soreness caused by their acne, which would make it difficult to get to sleep; for others itchiness made it difficult to sleep. Three participants sometimes found it difficult to sleep because they worried about their acne or felt depressed about it.

\section{Impact on School or Work}

All of the adolescent participants were at school or college; although most felt that their school work was not affected by acne, some mentioned feeling distracted by their acne when it was at its worst. Three participants contributed less to class activities because of the impact of acne on their self-confidence. A few participants were sometimes bullied or picked on at school about their acne. For two participants, acne affected them to the extent that they took days off school when their acne was at its worst.

Acne also affected some adult participants' work. Two participants felt they were less likely to get jobs because they would be judged on their acne, while another opted to work at home when his acne flared up under the pretense of having chickenpox. One participant 


\section{Example quotes from adolescents}

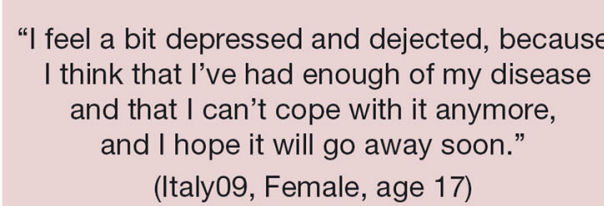

(Italy09, Female, age 17)

"I would just like to stay in bed and don't see anyone. I feel really bad on such a day. Sometimes I just cry, because I don't want all this anymore. That's what it is like on bad days."

(Germany08, Female, age 16)

"Like if I'm having a bad day and there's a party or something I don't go. So I sort of exclude myself from certain social situations. I'm not myself; I'm just quiet and like in the shadows a bit."

(UK20, Female, age 16)

"Well I use Instagram and posting selfies and stuff is not really something I enjoy doing because I look at my friends' selfies and I think they're actually brilliant and they don't have to worry about anything, when I post stuff I'm always thinking maybe people are going to screenshot it and search for their friends people and, I don't know, will just like judge me on my acne."

(UK05, Female, age 13)
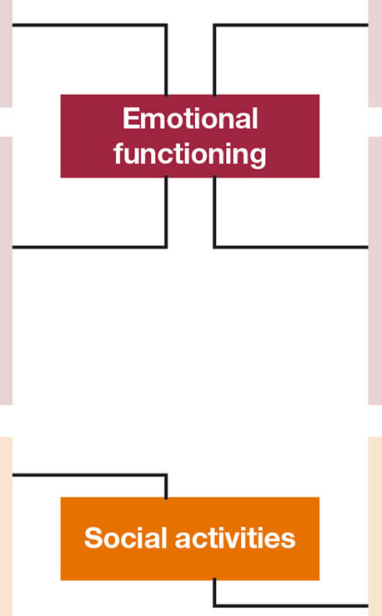

"Well when it's bad I won't go out; I won't go out if it's one of my friends' birthdays, I won't go out to clubs, I won't, you know if it's bad I won't go out."

(UK11, Female, age 29)

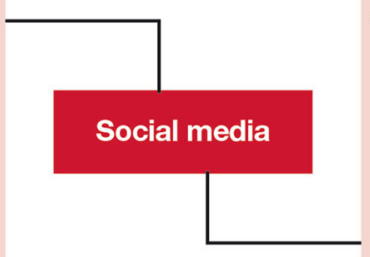

"I'm quite careful when I'm out and about that no one's taking anything close up. If anything does slip through the cracks I'll sort of send my friend an angry message asking them to take it off Facebook. I do get quite paranoid that a bad picture is going to appear."

(UK16, Female, age 19)

"People have split up with me because of how bad my acne got and that didn't do anything... it had a negative effect on my self-confidence and self-esteem."

(UK05, Male, age 23)

Fig. 2 Example quotes related to the impact of acne on emotional functioning, social activities, social media, and relationships

had lost her job because she would not go to work when her acne was bad, another participant had resigned from her job because she felt people were laughing at her acne. A student nurse felt patients thought he had an infection when his acne flared up and therefore did not want him to treat them.

\section{Perception of Acne/Reaction of Others}

Most participants had experienced people being mean or insensitive to them about their acne. For adults this was more common when they were younger and people were generally now more sympathetic. Many participants felt that 


\section{Example quotes from adolescents}

"I'm not really sporty because as I say I don't really go out as much; I used to be a lot more sporty but I kind of stopped going along.(...) I played for a team and I didn't really want to be going out as much and didn't want to be around them with acne." (UK06, Male, age 17)

"Yes, let us say that there are some periods when the acne is more severe and of course it's not pleasant, so you try to hide it. In such a situation, sometimes I don't feel like going out, and I try to do as less things as possible."

(Italy09, Female, age 17)

"Before I had the cream it affected it loads, like I would sometimes wake up with soreness and stuff and I couldn't get back to sleep."

(UK07, Female, age 13)
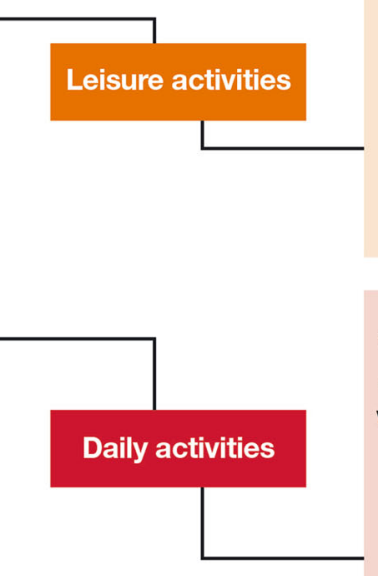

"It makes me feel embarrassed and awkward so I tend to try and go shopping or do things when people are at work or through the night, you know like the Tesco where I live is open

24 hours, I've been used to just going to Tesco at midnight."

(UK26, Male, age 45) all my massive scars and things like that on my back with it all on my shoulders. So I haven't been swimming for about the last 7 years or something like that, not even on holiday."

(UK14, Female, age 47)
"When it was really bad I just avoided school so yes my studies were really affected at one point (...) l'd say mom l'm feeling sick I'm not going to school." (UK20, Female, age 16)

\section{k}

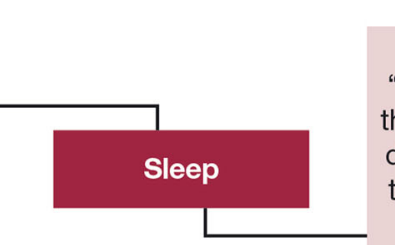

"I'll not go swimming because you've got to wear a swimming costume and you can see

"When I have bad acne I find it hard to sleep thinking about it. If I'm going to work the next day or going to see family or friends or going to work I find ways... I'm thinking of ways of avoiding it so that affects my sleep."

(UK27, Male, age 26)
"The fact that people think that you don't clean your face properly and stuff and people just judge you on your appearance especially nowadays you know where appearance is pretty much everything, you have to look all clean and nice and when people are just going to judge you." (UK07, Female, age 13)
School/work

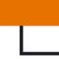

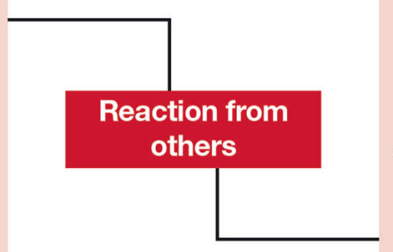

"One does not feel good and it makes me self-conscious. When someone looks at me for a while I think they only look at my spots and judge me because of that. One just feels queasy."

(Germany01, Female, age 19)

Fig. 3 Example quotes related to the impact of acne on leisure activities, daily activities, sleep, school/work, and reaction from others

people stared at them because of their acne; this made them feel self-conscious or nervous and affected their self-confidence. Some participants felt that they are judged on the basis of their acne and are viewed in a negative way because of it. 
Five adolescents and half of adults felt that people thought their acne must be their fault in some way; either caused by a lack of hygiene and not looking after their skin properly, a bad diet, or drinking alcohol.

Participants were asked whether they felt their acne is taken seriously by other people. Adolescents generally felt their acne is taken seriously, particularly by their family and their doctor. Over half of adults felt their acne is not taken seriously by others, including their general practitioner (GP); some had to visit their GP several times before they were referred to a dermatologist.

\section{Conceptual Model}

A conceptual model was developed from the qualitative data, which illustrates the impact of acne on HRQL and the links between concepts (Fig. 4). Emotional functioning is central to the conceptual model, as this appeared to have a subsequent impact on all other areas of HRQL.
The arrows in the model are based on the qualitative data where participants indicated that concepts are linked and indicate the direction of influence. The dotted arrows indicate a potential moderator that can have a positive or negative influence on the extent to which HRQL is impacted. A concept that was common throughout adolescent and adult interviews was the perception of acne from others and their reaction to it, which potentially had an impact on all areas of HRQL.

\section{Attributes of Topical Acne Treatments}

Participants were asked to rate how important several different acne treatment attributes are to them on a scale from "not important at all" to "critical/essential". The same three attributes were rated most highly by adolescents and adults: treatment works quickly, does not cause irritation, and does not bleach. Some example quotations about the treatment attributes are displayed in Fig. 5. For adolescents it was

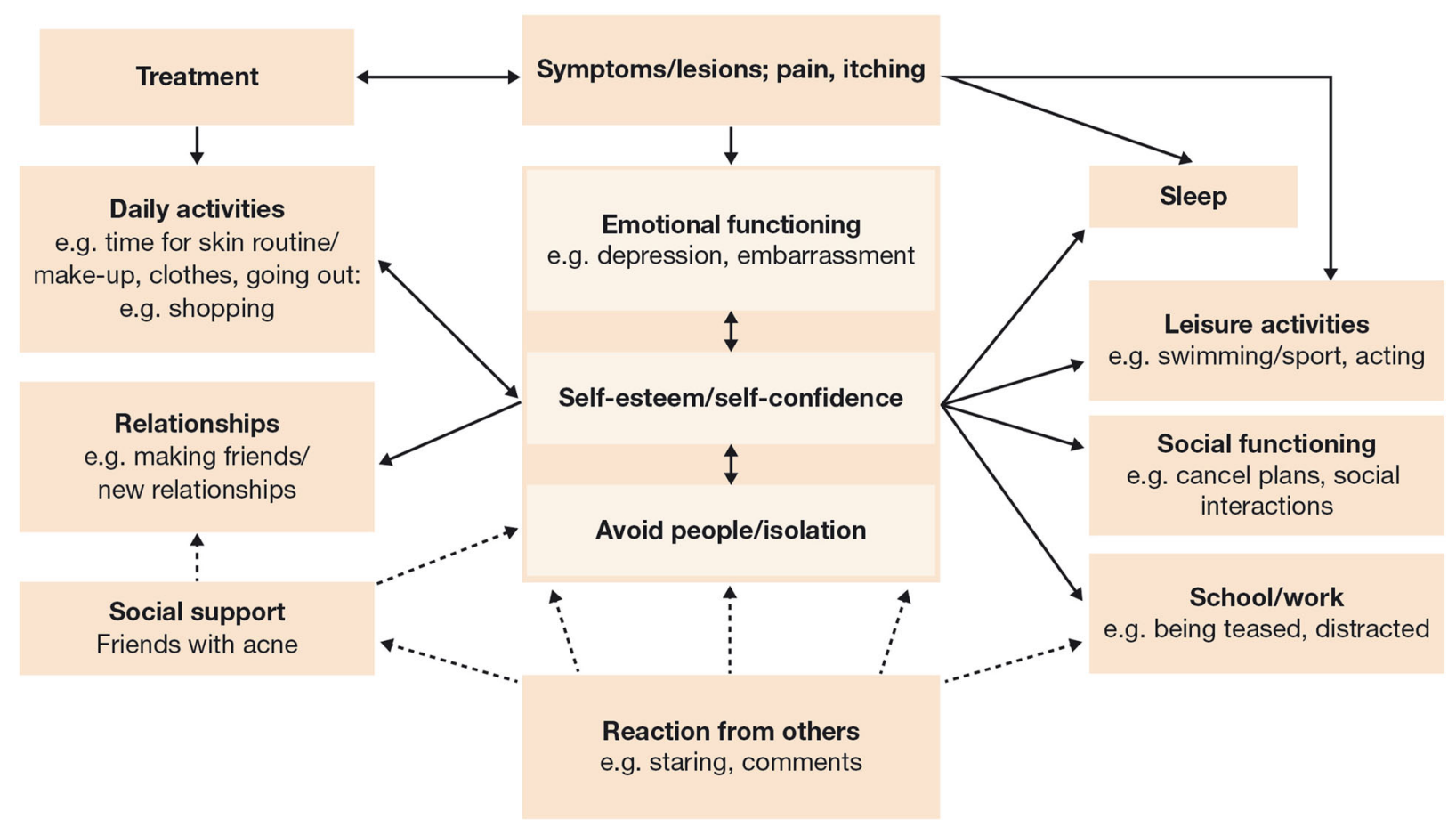

Fig. 4 Conceptual model of the impact of acne on healthrelated quality of life (HRQL). Arrows indicate direction of influence; dotted arrows indicate a potential moderator that can have a positive or negative influence on the extent of impact on HRQL. This figure was first presented at the 26th European Academy of Dermatology and Venereology Congress, September 13-17, 2017, Geneva, Switzerland 


\section{Example quotes from adolescents}

“Just so that maybe you don't have to spend a few months before you see any kind of improvement because it's still going to have a major affect in that time on your social life."

(UK25, Male, age 17)

\section{Example quotes from adults}

"Because if a product starts working very quickly, the consumer acquires trust in the product while if the product action is delayed in time, then the consumer tends not to trust that product and to stop using it even before it starts being effective." (Italy03, Male, age 18)

\footnotetext{
"You don't want your skin to be irritated when you already have the irritation by the acne so I don't want my skin to be like even more damaged by a cream."

(UK31, Female, age 16)
}

Works quickly

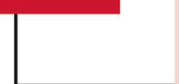

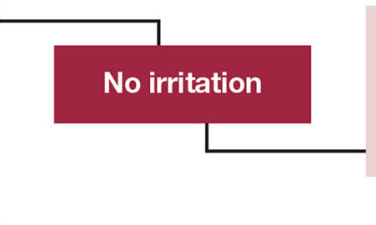

No irritation any further irritation, it would be uncomfortable."

(Italy04, Female, age 33)

\begin{abstract}
"Because the previous therapy I was using, would bleach clothes quite a lot, therefore it was very annoying, whereas this does not happen with my current therapy and this is important for me. In terms of impact on my life, I needed to apply my previous treatment before I went to sleep and, obviously, I used poor quality bed sheets, because the cream would bleach them." (Italy09, Female, age 17)
\end{abstract}

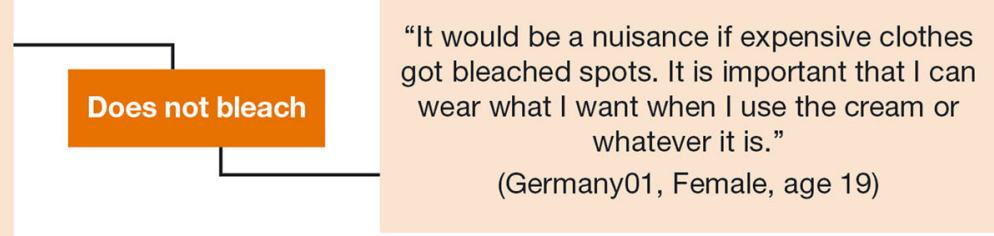

Fig. 5 Example quotes about the attributes of topical acne treatments

important that an acne treatment does not contain alcohol; several felt that alcohol might sting or irritate their skin and cause a burning sensation. This attribute was generally less important for adult participants who felt that the treatment ingredients were not important as long as the treatment was effective. Some participants felt it was very important for a treatment to only require application once a day; however, some participants would apply it as many times as necessary if it was effective. For some participants it was important that the treatment could be applied with their fingers rather than an applicator or pad as they felt it would be more hygienic and convenient. Similarly, for convenience, some participants preferred a treatment that did not need to be kept refrigerated.

\section{DISCUSSION}

This qualitative study explored the impact of acne and its treatment on adolescents' and adults' HRQL and found that HRQL was affected by acne for all except one participant. The conceptual model illustrates the impact on HRQL and demonstrates the importance of an effective treatment for acne to reduce this. Although the effect of acne on adolescents and adults is similar, there are some important differences. For adolescents, acne is more common among people the same age; therefore, many adolescents had friends with acne or other people at school had acne and thus they had some support from others who understood how it felt. For adults, some commented that they do not know anyone else their age who has acne and therefore felt alone and suggested that others do not understand how it feels. Adults also commented that people view acne as something that affects teenagers and therefore felt that people believed it might be due to them not looking after their skin, their diet, or another skin condition. 
One difference between male and female participants was evident in the use of makeup to conceal acne, which reinforces the use of adjuvant therapies to improve acne treatment outcomes [29, 30]. A small number of male participants had tried to conceal their acne with cosmetics in the past; however, this topic was largely discussed by female participants and for some the ability to conceal their acne made it easier to cope with.

This study also explored the importance of different attributes of topical acne treatments. For both adolescents and adults the most important attributes were fast-acting treatments that do not cause irritation and do not bleach clothing. Adolescents felt it was important for a treatment to not contain alcohol; however, this was less important for adults. Adults placed more importance on a treatment that had no specific storage requirements. Given the low adherence to acne therapies [15] (and the link between dissatisfaction with treatment and non-adherence [16]), when prescribing treatments it is important to consider the attributes that may lead to increased satisfaction with treatment.

The results of this study support the many other studies that demonstrate the negative impact of acne on HRQL [5-7]. A previous study found that for adults with acne, perceived stigma is a significant predictor of acne-related HRQL, contributing more than factors such as severity, gender, or age [31]. The findings of the current study provide qualitative support to this; although the term "stigma" was not used by participants, the negative impact of feeling judged by their acne or feeling as though people are staring at their acne was discussed by most participants.

Participants in the current study reported ways in which acne affects their use of social media. Although many participants had social media accounts, they did not post pictures of themselves if their acne was visible and avoided having pictures taken as they did not want them to be posted online by others. Previous studies have explored social media in relation to dermatology in terms of it being a source of information or support [32, 33]; however, to our knowledge, this is the first study to explore the impact of acne on use of social media.

This study illustrates the impact of acne through the development of a conceptual model from qualitative data. Conceptual models can be useful tools that provide a visual representation of the impact of a condition, allowing the links between concepts to be identified. In addition to highlighting the many areas of HRQL that are affected by acne, the model can be used to identify concepts of interest for future studies, to guide selection of an appropriate instrument to measure the impact on HRQL [23], or as the basis for the development of a new instrument to assess HRQL in acne. While existing acne-specific HRQL instruments assess several of the concepts identified in the conceptual model, there were concepts identified as important to participants in the current study that are not assessed by such instruments. For example, most instruments do not capture the impact of acne on sleep, daily activities, or work/school; therefore existing instruments may not fully capture the impact of acne on HRQL.

Participants discussed some ways in which their relationships are affected by acne. As reported in the results, some participants discussed feeling anxious or embarrassed when talking to new people or feeling like they were judged on their acne, which caused problems with forming relationships with girlfriends/ boyfriends. Although some participants discussed this, many did not report an impact on this area of their HRQL. It may be that adolescents in particular did not feel comfortable speaking about this or that a parent or family member was in the room during the interview, which made participants reluctant to discuss it. This is a possible limitation of the study, as qualitative telephone interviews may not have obtained the depth of data on this topic that might be expected. Items on relationships are included in several acne-specific instruments (Acne-QOL, AQOL, DLQI, Skindex29) and it may be that using quantitative methods is a more appropriate way to explore this concept.

Some limitations should be considered when interpreting the findings of this study. The 
extent to which the findings can be generalized to all adolescents and adults with acne is limited by the small sample size; however, data saturation was reached for both adolescent and adult samples, suggesting that additional interviews may not have introduced new concepts. The recruitment also relied on self-reported severity in order to identify people with moderate-severe acne; however, all participants were currently or recently prescribed topical treatment and had facial papules and/or pustules at the time of the interview. Although some participants experienced acne on several areas of their body, the inclusion criteria only required participants to have facial acne; therefore, the results can only be assumed to be relevant to facial acne. In addition, it is possible that experiences of older adolescents and young adults are similar and any differences noted between the adolescent and adult samples are really due to the older adults in the sample. The German adult sample consisted of teenagers; therefore, the experience of older adults in Germany may not be represented.

\section{CONCLUSIONS}

The results of this qualitative study demonstrate that moderate-severe acne has an extensive impact on adolescents' and adults' HRQL. The conceptual model illustrates the many areas of HRQL that are affected and draws attention to the importance of effective treatments for acne. The study highlights the importance of measuring HRQL in future interventional studies of acne treatments and identifies treatment attributes that are most important to patients.

\section{ACKNOWLEDGEMENTS}

Funding. This study and article processing charges were funded by Meda, a Mylan company. All authors had full access to all of the data in this study and take complete responsibility for the integrity of the data and accuracy of the data analysis.
Authorship. All named authors meet the International Committee of Medical Journal Editors (ICMJE) criteria for authorship for this manuscript and have given final approval of the version to be published.

Medical Writing and/or Editorial Assistance. Medical writing assistance in the preparation of this manuscript was provided by Jane Murphy (CircleScience, an Ashfield Company, part of UDG Healthcare plc) and funded by Meda, a Mylan company.

Thanking Patient Participants. Thank you to study participants for their involvement in this study.

Disclosures. Gabriella Fabbrocini receives research fees from Meda, a Mylan Company. Giuseppe Monfrecola receives research fees from Meda, a Mylan company. Sara Cacciapuoti has nothing to disclose.

Compliance with Ethics Guidelines. The research was designed to comply with guidelines for conducting research with children and young people [24]. Ethical approval was provided by Salus IRB, an independent review board, and the study was conducted in accordance with the principles of the Declaration of Helsinki. All adult participants provided informed consent prior to participating in the study; parents or guardians of adolescent participants provided informed consent for their child to participate; adolescents also provided assent.

Data Availability. The datasets generated and/or analyzed during the current study are available from the corresponding author on reasonable request.

Open Access. This article is distributed under the terms of the Creative Commons Attribution-NonCommercial 4.0 International License (http://creativecommons.org/licenses/ by-nc/4.0/), which permits any noncommercial use, distribution, and reproduction in any medium, provided you give appropriate credit to the original author(s) and the source, provide 
a link to the Creative Commons license, and indicate if changes were made.

\section{REFERENCES}

1. Tan JK, Bhate K. A global perspective on the epidemiology of acne. Br J Dermatol. 2015;172(Suppl 1):3-12.

2. Smithard A, Glazebrook C, Williams HC. Acne prevalence, knowledge about acne and psychological morbidity in mid-adolescence: a communitybased study. Br J Dermatol. 2001;145(2):274-9.

3. Williams HC, Dellavalle RP, Garner S. Acne vulgaris. Lancet. 2012;379(9813):361-72.

4. Gollnick H, Cunliffe W, Berson D, et al. Management of acne: a report from a global alliance to improve outcomes in acne. J Am Acad Dermatol. 2003;49(1 Suppl):S1-37.

5. Klassen AF, Newton JN, Mallon E. Measuring quality of life in people referred for specialist care of acne: comparing generic and disease-specific measures. J Am Acad Dermatol. 2000;43(2 Pt 1):229-33.

6. Mallon E, Newton JN, Klassen A, Stewart-Brown SL, Ryan TJ, Finlay AY. The quality of life in acne: a comparison with general medical conditions using generic questionnaires. $\mathrm{Br} \mathrm{J}$ Dermatol. 1999;140(4):672-6.

7. Tasoula E, Gregoriou S, Chalikias J, et al. The impact of acne vulgaris on quality of life and psychic health in young adolescents in Greece. Results of a population survey. An Bras Dermatol. 2012;87(6):862-9.

8. Cresce ND, Davis SA, Huang WW, Feldman SR. The quality of life impact of acne and rosacea compared to other major medical conditions. J Drugs Dermatol. 2014;13(6):692-7.

9. Kellett SC, Gawkrodger DJ. The psychological and emotional impact of acne and the effect of treatment with isotretinoin. $\mathrm{Br} \mathrm{J}$ Dermatol. 1999;140(2):273-82.

10. Dalgard F, Gieler U, Holm JO, Bjertness E, Hauser S. Self-esteem and body satisfaction among late adolescents with acne: results from a population survey. J Am Acad Dermatol. 2008;59(5):746-51.

11. Magin P, Adams J, Heading G, Pond D, Smith W. Psychological sequelae of acne vulgaris: results of a qualitative study. Can Fam Phys. 2006;52:978-9.

12. Loney $\mathrm{T}$, Standage M, Lewis S. Not just 'skin deep': psychosocial effects of dermatological-related social anxiety in a sample of acne patients. J Health Psychol. 2008;13(1):47-54.

13. Smith JA. The impact of skin disease on the quality of life of adolescents. Adolesc Med. 2001;12(2):343-53.

14. Thiboutot D, Gollnick H, Bettoli V, et al. New insights into the management of acne: an update from the Global Alliance to Improve Outcomes in Acne group. J Am Acad Dermatol. 2009;60(5 Suppl):S1-50.

15. Dreno B, Thiboutot D, Gollnick H, et al. Large-scale worldwide observational study of adherence with acne therapy. Int J Dermatol. 2010;49(4):448-56.

16. Renzi C, Abeni D, Picardi A, et al. Factors associated with patient satisfaction with care among dermatological outpatients. $\mathrm{Br} \mathrm{J}$ Dermatol. 2001;145(4):617-23.

17. Kellett N, West F, Finlay AY. Conjoint analysis: a novel, rigorous tool for determining patient preferences for topical antibiotic treatment for acne. A randomised controlled trial. $\mathrm{Br} \mathrm{J}$ Dermatol. 2006;154(3):524-32.

18. Girman CJ, Hartmaier S, Thiboutot D, et al. Evaluating health-related quality of life in patients with facial acne: development of a self-administered questionnaire for clinical trials. Qual Life Res. 1996;5(5):481-90.

19. Finlay AY, Khan GK. Dermatology Life Quality Index (DLQI) - a simple practical measure for routine clinical use. Clin Exp Dermatol. 1994;19(3):210-6.

20. Gupta MA, Johnson AM, Gupta AK. The development of an acne quality of life scale: reliability, validity, and relation to subjective acne severity in mild to moderate acne vulgaris. Acta Derm Venereol. 1998;78(6):451-6.

21. Magin P, Heading G, Adams J, Pond D. Sex and the skin: a qualitative study of patients with acne, psoriasis and atopic eczema. Psychol Health Med. 2010;15(4):454-62.

22. Murray CD, Rhodes K. 'Nobody likes damaged goods': the experience of adult visible acne. $\mathrm{Br} \mathrm{J}$ Health Psychol. 2005;10(Pt 2):183-202.

23. Rothman ML, Beltran P, Cappelleri JC, Lipscomb J, Teschendorf B. Patient-reported outcomes: conceptual issues. Value Health. 2007;10(Suppl 2):S66-75.

24. MRS guidelines for research with children and young people (internet). (September 2014). https:// www.mrs.org.uk/pdf/2014-09-01Children\% 
20and\%20Young\%20People\%20Research\%

20Guidelines.pdf. Accessed 11 July 2017.

25. Sturges JE, Hanrahan KJ. Comparing telephone and face-to-face qualitative interviewing: a research note. Qual Res. 2004;4:107-18.

26. Vogl S. Telephone versus face-to-face interviews mode effect on semistructured interviews with children. Sociol Methodol. 2013;43:133-77.

27. Braun V, Clarke V. Using thematic analysis in psychology. Qual Res Psychol. 2006;3:77-101.

28. Morse JM. The significance of saturation. Qual Health Res. 1995;5:147-9.

29. Araviiskaia E, Dreno B. The role of topical dermocosmetics in acne vulgaris. J Eur Acad Dermatol Venereol. 2016;30(6):926-35.
30. Del Rosso JQ. The role of skin care as an integral component in the management of acne vulgaris: part 1: the importance of cleanser and moisturizer ingredients, design, and product selection. J Clin Aesthet Dermatol. 2013;6(12):19-27.

31. Liasides J, Apergi FS. Predictors of quality of life in adults with acne: the contribution of perceived stigma. EpSBS. 2015. https://doi.org/10.15405/ epsbs.2015.01.17 (II:icCSBs).

32. Savas JA, Huang KE, Tuchayi SM, Feldman SR. Understanding the influence of social media in medicine: lesson learned from Facebook. Dermatol Online J. 2014;20(9). pii: 13030/qt2099j0z1.

33. Whitsitt J, Mattis D, Hernandez M, Kollipara R, Dellavalle RP. Dermatology on Pinterest. Dermatol Online J. 2015;21(1). pii: 13030/qt7dj4267p. 